\title{
WAIWAI: UMA DESCRIÇÃO MÍNIMA À HISTÓRIA DO CONTATO ${ }^{1}$
}

\author{
Jorge Manoel COSTA E SOUZA ${ }^{2}$
}

\begin{abstract}
RESUMO: Os Waiwai, índios de filiação karib, habitam atualmente aldeias localizadas ao norte do Brasil, nos Estados do Pará e Roraima, além do grupo localizado no rio Essequibo, na República Cooperativista da Guiana. A população atual dos Waiwai é superior a duas mil pessoas. Neste artigo, relato o processo de contato do grupo com a sociedade ocidental, em especial, a abordagem realizada por pesquisadores e estudiosos da questão indígena. O tema permite conhecer a origem da etnografia waiwai, a participação e a influência dos missionários entre esta sociedade, a movimentação dos grupos locais e, por fim, conseqüências advindas do contato.
\end{abstract}

Palavras-chave: Waiwai, Amazônia, índios, contato, etnografia, migração, aldeia, etnicidade.

\section{Waiwai: A little description at contact history.}

ABSTRACT: Waiwai, Indian of filiation karib, inhabit nowadays villages located in the north of Brazil, in state lands of Pará and Roraima, besides the group located in the river Essequibo, in Guiana. Waiwai's current population is superior to two thousand people. In this paper report the contact process of the group with the western society, especially, the boarding accomplished for indigenous matter searching and studious. The theme allows to know the ethnography waiwai origin, the participation and missionaries' influence in this society, the movement of the local groups and, finally, the consequences and effects of the contact.

Key-Words: Waiwai, Amazonian, Indian, contact, ethnography, migration, village, etnicidade.

${ }^{1}$ Comunicação produzida para ser comentada no encontro promovido pela Fundação Nacional de Saúde (FUNASA), por ocasião da organização do Distrito Sanitário Leste de Roraima, em 14/08/2000, em Boa Vista.

2 Mestre em Antropologia Social, Setor de Antropologia/Museu Integrado de Roraima - MIRR Av. Brigadeiro Eduardo Gomes s/n Parque Anauá 69305-010 Boa Vista, Roraima, Brasil.

Nota do autor: Agradeço à Professora, Dra. Alcida Rita Ramos (UnB) por ter realizado uma leitura preliminar deste artigo. 
Este artigo alude acerca da história do contato que conduziu a sociedade indígena waiwai à interface permanente e crescente de envolvimento com o mundo dos brancos. Em particular, tento resenhar o encontro dos Waiwai com pesquisadores e exploradores europeus que excursionaram pela chamada área cultural das guianas (Rivière, 1984), a partir do século XIX. Esse tipo de contato possui perfil próprio. Ele permitiu produzir as primeiras referências ocidentais do grupo. Tais observações evoluíram da forma incipiente de relatos de viagens à condição de narrativas etnográficas, base de construção de etnografias propriamente ditas. o decorrer deste século proporcionou a pesquisadores da América do Norte, da América Central e da América do sul uma investigação razoável a respeito do grupo. Trabalhos etnográficos realizados por pesquisadores brasileiros entre os Waiwai ainda podem ser dados como tímidos. Poder-se-ia afirmar que tais estudos contemplam mais fins estatísticos e menos etnológicos, haja vista suas características fragmentárias, portanto, resumidos como contribuição significativa à extensão do corpo etnológico literário produzido pela antropologia brasileira. Ainda que "ausentes" da tradição etnológica nacional, os Waiwai representam um expoente étnico e social na Amazônia oriental brasileira do final do século XX (Costa e Souza, 1988). Os Waiwai pertencem à família lingüística karib - hoje possuem uma população superior a 2 mil pessoas -, vivem em sua maioria em um aldeamento chamado na língua de Yxamna com alta concentração demográfica, e em outras três principais malocas (Anauá, Titkoñeri e Aaku), com populações que oscilam entre 100 e 250 pessoas. São aldeias que contrastam com os padrões de suas tradicionais habitações (mî̂mo) e as formas primordiais da organização social do grupo. Em oposição à ocupação dispersa de ambientes da floresta e a alta mobilidade característica dos grupos locais ancestrais, atualmente os Waiwai desenvolveram núcleos comunitários de pouca mobilidade no estado de Roraima e ao norte do estado do Pará, além do grupo local do alto Essequibo da Guiana, este último ocupante de uma parte do território ancestral setentrional.

A sociedade majoritária, em especial à dos regionais e as politicas desenvolvimentistas locais, projeta expectativas de que os Waiwai muito brevemente engajarse-ão às relações de produção econômica e sociais periféricas, a exemplo do que ocorreu aos Makuxi de Roraima, de modo semelhante àquele relatado em Diniz (1972). No entanto, penso que tal projeção é equivocada. Tal expectativa ignora uma condição êmíca waiwai fundamental: o ethos waiwai. Ele envolve, além de fundamentos cosmológicos, a tradição da exclusão e inclusão condicionantes, características muito fortes da etnicidade e da fronteira étnica perseguidas do grupo em relação aos não-índios. Nesse sentido, há que se perceber uma reunião de contingências e condições identitárias razoavelmente definidas e orientadas por uma política social elaborada pelos Waiwai que, de certa forma, gera parâmetros aos vínculos de aproximação deles ao mundo dos brancos.

$\mathrm{Na}$ década de 1890 os Waiwai estavam quase extintos. Após esse período, seu reconhecimento étnicoidentitário decorreu de um longo processo de assimilação e intercasamentos iniciados primordialmente com parucoto e tarumã. Nos últimos trinta anos os Waiwai assumiram a hegemonia 
Waiwai: Uma descrição mínima à história do contato.

ante seus vizinhos étnicos. Construíram para si um status que atribui grau de importância ao grupo, ao mesmo tempo em que faz dele o caminho para o conhecimento da região, da interface do contato com outros povos internados na floresta e envolvidos ou perseguidos pelos Waiwai no seu intermitente processo de atração. o processo de assimilação de tríbos como karafawyana, hiskariana, xereu, mawayana, katuena, tiryo, entre outras, pelos Waiwai pode ser entendido como um fator de catarse (Howard, 1993: 257-59). Não é um processo explicado através da incursão belicosa ou guerreira. Diferentemente do que costuma acontecer entre grupos vizinhos arredios ou, por exemplo, os waimiri-atroari (cf. Baines, 1990; Sabatini, 1988 ) envolvidos historicamente em tradições guerreiras. Os Waiwai costumam utilizar-se da persuasão estética e do status que lhes são atribuídos para persuadir seus vizinhos. Há décadas eles vivenciam longos períodos não marcados por enfrentamentos guerreiros ou litigiosos.

\section{Relatos de viagens e etnografia}

A revelação da existência de três aldeias waiwai em 1837 por $\mathrm{H}$. R. Schomburgk (Fock, 1963: 5, 7), parece se constituir no primeiro dado informando da presença waiwai na região da serra Acaraí que, a julgar pelas informações históricas, pode ser caracterizada como verdadeiro território ancestral do grupo, reconhecendo o alto curso do rio Mapuera e formadores (porção meridional); o alto curso do Essequibo e as drenagens adjacentes (porção setentrional). ${ }^{3}$
Tais marcos referenciais, entretanto, parecem nunca ter delimitado a movimentação do grupo. os Waiwai efetivamente estão envolvidos em uma vasta rede de comércio. Assinalada pela interetnicidade, a rede baseia-se em trocas diversas. O exercício dessa atividade atinge grande área das Guianas e envolve uma complexidade de relações (Dreyfus, 1993 ).

Henri, A. Coudreau contatou os Waiwai em 1884, quando produziu descrições a respeito do grupo estabelecido na serra Acaraí, no alto rio Mapuera, em seus afluentes e no Essequibo. William C. Farabee esteve entre os Waiwai na região Acaraí em 1913-14, cujas pesquisas são publicadas em The Central Caribs (1924). Farabee descreve a ocupação waiwai em ambos os lados da montanha. Walter E. Roth, em 1925, encontrou-se com os Waiwai que viviam junto ao Essequibo, resultado de um movimento que os teria deslocado do Mapuera para aquela região por volta de 1920. A expedição de Terry-Holden (1937) relata que os Waiwai advindos do Brasil para a Guiana se instalaram nas proximidades de Yakayaka, abrigando-se em duas aldeias (Holden, 1938: 239; Fock, 1963: $8)$. Os dados de Terry-Holden identificam ainda quatro aldeias ao sul da montanha Acaraí, nas adjacências de rios formadores do Mapuera, mais especificamente acima do encontro do rio Urukurin (Urucurina) com aquele. Braz Dias de Aguiar, chefe da Comissão Brasileira Demarcadora de Limites (1930-40), faz referências à presença de pequenas malocas waiwai nas terras fronteiriças do Brasil e da Guiana Inglesa, em ambas as faces da serra Acaraí. Os trabalhos da Comissão anglo-brasileira,

A região norte da serra Acarai pertence à República Cooperativista da Guiana; a região sul, pertence ao Brasil, e se estende pelo médio e alto cursos do rio Mapuera e seus formadores, compreendendo áreas a sudeste de Roraima e norte do Pará.

Bol. Mus. Integrado de Roraima, Boa Vista 5:3-12. 1999 
merecem uma menção rápida de Fock (1963: 8). Wai-Wai, publicado por Nicholas Guppy em 1958, reúne descrições aterradoras das condições epidêmicas e lastimáveis vividas pelos Waiwai do lado meridional da serra Acaraí, precisamente no alto Mapuera. É uma espécie de relatório do pavor, do medo e do terror ocasionados pelo contato, à moda Taussig em Xamanismo, Colonialismo e o Homem Selvagem do vale colombiano do Putumayo (Taussig, 1993 ).

Estudos da imemorialidade da ocupação waiwai no Brasil, cosmologia, organização social, xamanismo, cultura material, etc. devem ser considerados nos dados etnográficos presentes nos trabalhos de Niels Fock: Waiwai. Religion and Society of an Amazonian Tribe (1963), e de J. Yde em Material Culture of the Waiwai (1965). Admito serem esses os trabalhos mais amplos e abrangentes a respeito do grupo. Ambos os autores pertencentes a uma equipe de etnógrafos do Danish National Museum, estiveram em visita aos Waiwai entre agosto de 1954 e janeiro do ano seguinte; entre agosto e dezembro de 1958 (CEDI, 1983, v.3 :233-40; Queiroz, 1996: 215). Uma outra fonte interessante para conhecer a trajetória waiwai foi escrita pelo repórter estadunidense Homer E. Dowdy (1963, editora Harper and Row) intitulada Christ's Witchdoctor. Permite conhecer facetas da relação dos missionários da Univangelized Fields Mission - UFM ${ }^{4}$ com o grupo, assim como traz informações que facilitam reconhecer os antigos limites do território ancestral e de perambulação efetiva dos Waiwai. "Wai-Wai", artigo publicado pela FUNAI na revista "Atualidade
Indígena" (FUNAI, 1978: 42-4), traz relatos ligeiros de sertanistas conhecedores da região, entre eles, Otávio Pinheiro Canguçu, que atuou entre os Waiwai, e Gilberto Pinto Figueiredo, responsável pela frente de atração waimiri-atroari. No mesmo artigo é narrado, pelo antropólogo Célio Horst, um pouco da história a respeito da conversão e da saga de Ewka, o grande xamã waiwai, tido como principal responsável pela conversão do grupo ao cristianismo. Também discorre sobre a situação dos Waiwai do rio Anauá da aldeia Caxmi. Individual and Society in Guiana (1984) de Rivière, me parece ser - que há de mais aglutinador em se tratando de resenhas acerca da literatura antropológica já elaborada e enfocando temas relacionados a grupos waiwai locais contemporâneos. 'Pawana: a Farsa dos "Visitantes" entre os Waiwai da Amazônia Setentrional' (1993), é um artigo escrito por C. Howard (Universidade de Chicago e Museu Nacional do Rio de Janeiro). Howard trata da vocação waiwai no processo de assimilação e contato intertribal, da tradicional festa de waiwaização da pessoa envolvendo a farsa, a paródia, a pantomima, a dramatização..., além de explorar argumentos que levaram uma facção do grupo à construção do aldeamento do rio Jatapuzinho, ajudados por parentes estabelecidos nas margens do rio Anauá e afluentes. Explora, em especial, a chamada construção do sentido envolvendo a waiwaização da pessoa e a noção de tribo, ao estabelecer homologias entre caricaturas socializantes e o mundo cosmológico do grupo. ${ }^{5}$ Estudos relacionados ao parentesco, à organização social

4 Traduzida como "Missão para os Campos não Evangelizados".

5 C. Howard defendeu recentemente (nos Estados Unidos) tese de doutoramento cujo tema central envolve os Waiwai, entretanto, até a data de elaboração desta nota não tive acesso ao referido trabalho. 
Waiwai: Uma descrição mínima à história do contato.

e a relações de gênero entre os Waiwai podem ser vistos em G. Mentore (1983-4). Mentore é hábil ao explorar tais características da vida social waiwai. Investigações etnográficas de igual importância considerando aspectos diversos do grupo são encontradas em P. Frikel (1957), Evans \& Meggers (1960-4, 1971), R. Dogon (1967), J. Morton (1979) e Queiroz (1996). Atualmente C. Machado (Universidade de São Paulo) desenvolve pesquisa entre os Waiwai de Titkoñeri (Jatapuzinho). o contato missionário e a
movimentação transnacional waiwai

A imigração massiva dos Waiwai para a ex-Guiana Inglesa teve início na primeira metade dos anos cinqüenta com a instalação da "Missão Canashen", no Essequibo. Mantida pela Univangelized Fields Mission, com sede em Baltimore, nos Estados Unidos (Dowdy, 1997: nt), a Missão não perece ter sofrido impedimentos maiores que dificultassem a atração e a imigração de índios que habitavam a calha dos rios Mapuera, Trombetas, Nhamundá e Paru de Oeste para a Guiana. Muito pelo contrário, a política indigenista brasileira até colaborou, à medida que impunha um estado de abandono aos índios, largando-os à própria sorte; por outro lado, a persuasão missionária estabelecida no alto Essequibo convencia os índios de que Canashen era melhor para eles. O êxodo contínuo das populações indígenas do Mapuera, Trombetas, Nhamundá e de seus formadores ensejou o envio de uma comissão militar brasileira à região de fronteira para averiguar a situação. Em 1959 ocorreu uma importante expedição de interesse militar, do Serviço de Proteção aos Índios - SPI e dos religiosos franciscanos da Sociedade dos $\mathrm{Pa}-$ dres Missionários da Prelazia de óbidos que, juntos, estabeleceram a "Missão Erepecuru" entre os tiryó no alto rio Paru de Oeste, na fronteira do Brasil com o Suriname. Chamada "Operação Mapuera", reconhecida como operação Mapex, a comissão visitou a região ao final do ano de 1962 e março do ano seguinte, comandada pelo Brigadeiro João Camarão Telles Ribeiro. O resultado do encontro entre a Comissão e a Missão em Canashen não inverteu nem modificou a situação de "fuga" dos índios "brasileiros" para a Guiana, à época motivados por uma proposta missionária salvacionista. A expedição do Brigadeiro João Camarão Teles Ribeiro ("Brigadeiro Camarão" como era conhecido pelos índios da região, denotado por sua filosofia e ética durante contatos, organizador dos chamados trinômios FAB, índios, missão) não obteve sucesso nessa empreitada, por conta da já citada malograda política nacional indigenista (cf. CEDI, 1983, V. 3: 190,91-233).

A 'Missão entre os Waiwai', instalada na Guiana, reuniu os Waiwai por quase 20 anos. Empenhouse em formar lideranças espirituais que influenciariam definitivamente os rumos futuros do grupo. Lideranças como as de Ewka, Yukuma, Mawaxa, Yakuta, Kirpaka, Xexeua, Tamokrana, foram "preparadas" ao longo desse tempo para fazer do povo Waiwai uma nação de "crentes" por excelência, com um propósito: contatar e converter outros grupos indígenas dispersos pela Amazônia oriental, tomados pelos waiwai como tirwoñe (ferozes e irados), carentes de civilidade e da verdadeira condição humana. Os Waiwai utilizaram-se do acesso privilegiado aos bens de troca oferecidos pelos missionários, do conhecimento da escrita, da terapia médica ocidental, entre outros e assumiram a eminência dentro do sistema regional aumentando, desta forma, rapidamente a dimensão de seus aldeamentos compósitos (Howard, 1993: 229-235; Frikel, 
1970: 29-30, citado em CEDI: 1983 ; Guppy 1958: 19-20, citado em CEDI: 1983).

Em 1971, em conseqüência do movimento revolucionário ocorrido entre os anos de 1968/69, e o abandono do interesse inglês pela ex-Colônia, manifestado formalmente a partir de 1966, o governo de tendências socialistas instalado na Guiana inviabilizou a permanência da Missão no interior do país (CEDI, 1983, v.3: 234 ). Uns poucos índios permaneceram no Essequibo, a maioria envolveu-se em um outro movimento migratório de volta ao Brasil, dispersando-se através do antigo território onde tradicionalmente viviam. O Suriname acolheu parte desse contingente, vez que também havia contribuído para o primeiro processo migratório que levou à construção de Canashen, com populações de tiriyo. Os Waiwai regressaram para o Pará e Roraima. (CEDI, 1983, V.3: 234-8). A movimentação dos índios foi seguida pelos missionários que se dividiram e optaram por integrar a organização missionária Missão Evangélica da Amazônia - MEVA, estabelecida em Boa Vista, e à Missão Cristã Evangélica do Brasil - MICEB, a qual se estabeleceu no Mapuera, em 1976 (id.). Grupos waiwai passaram então a empreender expedições à procura de índios isolados para contatá-los e evangelizá-los. Vasculharam o Nhamundá, o vale do Jatapu, regiões do Anauá e rio Novo. As expedições indígenas lideradas por Ewka contataram, principalmente, os karafawyana autocontidos no território de influência dos
Waiwai, quando algumas famílias foram conduzidas para a aldeia Yxamná ou Mapuera, no médio curso do rio do mesmo nome.

O estabelecimento das duas principais aldeias waiwai numa distância aproximada de $300 \mathrm{~km} \mathrm{em}$ linha reta, no sentido sulNoroeste, onde Yxamná está ao sul, no estado do Pará e Kaxmi a noroeste, em Roraima, no rio Novo, afluente do Anauá (cf. FUNAI, 1978; CEDI, 1983, v.3) fez crescer a movimentação waiwai na área. A partir de 1981 os Waiwai do Mapuera passaram a utilizar com maior freqüência esse espaço territorial ao empreenderem suas longas viagens que chegam a durar semanas. O itinerário dessas viagens no interior do território descreve uma trajetória sinuosa cujo curso se confunde com a sinuosidade dos igarapés Itxawau, Yukutu; dos rios Baracuxi, Jatapuzinho Jatapu; veredas abertas da BR 210 e, por fim, o rio Novo (Id).

A experiência waiwai em Canashen, que concentrou a demografia de grupos domésticos, contrária aos padrões tradicionais de ocupação da floresta e a ecologia do grupo, intensificou a pulverização de sua organização social, já atingida pelas amplas alianças de casamentos interétnicos, desde quando os Waiwai espalharam-se em pequenos grupos localizados ao longo das bacias do Trombetas, Mapuera e Nhamundá. As grandes aldeias compósitas pós-contato concentraram-se em locais estratégicos, entretanto, sem a devida assistência do órgão oficial e sem o acompanhamento missionário

\footnotetext{
${ }^{6}$ Trata-se de um movimento armado, de orientação política contrária ao modelo socialista perseguido ou sugerido pelo governo local para o país à época. O movimento armado foi protagonizado por um pequeno grupo de famílias brancas imigrantes ou de ascendência européia, residente em Lethem e arredores. Ideologicamente o grupo supunha que o governo de Gerogetown estatizaria a propriedade, confiscaria bens particulares e hostilizaria a iniciativa privada. O movimento foi esmagado pelo governo, seus mentores refugiaram-se na Venezuela e no Brasil e suas propriedades e riquezas foram apropriadas pelo Estado.
} 


\section{Waiwai: Uma descrição mínima à história do contato.}

diário ao qual estavam submetidos nos anos seguintes à imigração para a Guiana. Conclui-se que a forma de ocupação do território modificou-se profundamente, em decorrência da experiência vivida nos aldeamentos artificiais. Inúmeras características que orientavam a vida social, religiosa, cultural e políticoeconômica organizada nos settlements "primitivos" desapareceram. Os Waiwai como grupo têm crescido em população e em participação na economia informal a que estão sujeitos nas suas relações de troca, comércio e serviços com o mundo dos brancos, mas estão longe de assimilar ditas relações como configurações avançadas de suas sociedades. Expressam tão-somente uma experiência ou fase pronunciada de sua história do contato. Trata-se de uma experiência de trocas simbólicas e reais. Uma marcante interface do contato, explicitada na forma de interagir em uma fronteira de tensão permanente e excludente. O processo que se estabelece nesta relação tensa on border "prepara" o Waiwai que se sente atraído irresistivelmente pelo mundo dos brancos (percebe que não há equivalências ou homologia no plano socioeconômico entre ambas as sociedades). Sérios abalos têm recaído sobre a estrutura da organização social política e econômica waiwai. Induzidos pelos brancos e tentados pela necessidade, os Waiwai introduziram outras práticas relacionadas à obtenção de recursos compensatórios e coadjuvantes à sua economia baseada na horticultura, complementada através da caça e da pesca, além das coletas sazonais (Costa e Souza, 1988).

\section{Conseqüências e assimilações decorrentes do contato}

Analisando o conjunto de influências e interações que afetaram o ethos e a vida tradicional dos Waiwai a partir do início dos anos cinqüenta, período de maior ampliação dos níveis do contato é possível, de maneira sintética, relacionar uma seqüência de mudanças: 1) a troca da habitação comunal ocupada pelo epeka (grupos de irmãos) para o modelo ocidental da casa ocupada pela família nuclear; 2) a anulação da figura política e espiritual do xamã, fundamentada e diretamente relacionada ao sistema de crenças e da própria cosmologia do grupo, substituído pelos kamuñimune (pastores índios), imbuídos da reorganização da vida social na aldeia, através da criação de conselhos (instituição compostas de Kamuñimune) que deliberam acerca das regras sociais, considerando a doutrina e a moral missionadas; 3) o surgimento de grandes aldeias compósitas, o que envolve o grupo local numa preocupação extra, a da escassez precoce das potencialidades e dos recursos naturais dispostos à sua volta, considerando também a dependência do grupo aos produtos originários da sociedade ocidental; 4) a introdução irreversível de instrumentos e artefatos culturalmente desenvolvidos pela sociedade ocidental, e a fomentação irresistível às coisas da modernidade (motores de popa, motosserras, indumentáría, utensilios de pesca, arma de fogo, tv e vídeo, e outros hábitos alimentares incluindo o açúcar e - sal; 5) a sustação da exibição de rituais e festas manifestas na tradição cosmológica e mítica, como as festas, danças e rituais de significado profundo para o sistema simbólico do grupo, e a proibição conseqüente da ingestão de bebidas fermentadas utilizadas nos festivais; 6) a supressão da mitologia e da etnociência waiwai, entre os "convertidos", e a dependência aos conhecimentos ocidentais como os da medicina, 
por exemplo; 7) a interferência nas regras tradicionais do sistema de parentesco, modelos tradicionais de união entre casais; 8) a proibição dos tabus e evitações relacionados à dieta; 9) a memória, por parte dos índios, do vigor da política protestante anti-fetichista (cf Costa e Souza, 1988: 188-9; Fock, 1963: 126; Queiroz, 1996: 225; CEDI, 1983, V.3: 233).

Ademais, como diz Calavia Saéz (1998: 1-30) "... se já falamos em 'encontro de cosmologias' e 'encontro de sociologias' poderíamos qualificar o acontecido entre o MNTB e os Wai-wai como um 'encontro de políticas' (...) 'Índios crentes' ou 'índios missionários', os Wai-wai têm achado na nova religião um meio de arcabouçar uma sociedade mais ampla, e para definir um lugar no desconcerto interétnico do norte amazônico" .

\section{BIBLIOGRAFIA}

AGUIAR, Braz Dias de. (1940) 1943. "Nas fronteiras da Venezuela e Guianas britânica e neerlandeza". Separata dos anais do $9^{\circ}$ Congresso Brasileiro de Geografia. Rio de Janeiro: IBGE.

BAINES, Stephen G. 1993. "O território dos Waimiri-Atroari e indigenismo empresarial" . Universidade de Brasilia. Série Antropologia, n. 138.

CALAVIA SÁEZ, Oscar. 1998. "Campo religioso e grupos indígenas no Brasil": Universidade Federal de Santa Catarina. Col. Antropologia em Primeira Mão: (25).

CEDI (Centro Ecumênico de Documentação e Informação). 1983. "Índios do Nhamundá/Mapuera". In: C. A. Ricardo (org.), Povos indígenas no
Brasil. São Paulo: v. 3, p. 225249 .

CIDER (Centro de Informação da Diocese de Roraima). 1989. Indios de Roraima: Makuxi, Taurepang, Ingarikó, Wapixana. (E. Amodio \& V. Pira orgs.). Col. HistóricoAntropológica, 1.

COSTA E SOUZA, Jorge M. 1994. "Observações preliminares obtidas em visita à Usina Hidrelétrica do Alto rio Jatapu. Boa Vista: Museu Integrado de Roraima. Relatório.

- 1998. Os Waiwai de jatapuzinho e o irresistível apelo à modernidade. Florianópolis: Universidade Federal de Santa Catarina. Dissertação de Mestrado.

DINIZ, Edson Soares. 1972. Os Índios makuxi do Roraima: sua instalação na sociedade nacional. São Paulo: Universidade de Marília. Tese de Doutorado.

DOWDY, Homer E. 1997. O pajé de Cristo: uma história do amor de Deus operando milagres nas selvas da Amazônia. Título original: Christ's witchdoctor. New York: Harper \& Row, 1963; [Trad. Fausto Camargo César]. São Paulo: Editora Sepal.

DREYFUS, Simone. $1993 . \quad$ "Os empreendimentos coloniais e os espaços políticos indígenas no interior da Guiana ocidental (entre - Orenoco e o Corentino) de 1613 a 1796". In: E. Viveiros de Castro \& M. Carneiro da Cunha (orgs.), Amazônia: etnologia e história indígena. São Paulo: NHIIUSP : FAPESP.

EVANS, C. \& MEGGERS, B. J. 1960. "Archeological investigations in British Guiana". In: Bulletin of the American Ethnology (117). Washington: Smithisonian Institution.

FARABEE, William C. 1924. The central Caribs. University of Pennsylvania. Philadelphia: Anthropological Publications, vol. 10. 
Waiwai: Uma descrição mínima à história do contato.

FARAGE, Nádia. 1991. As muralhas dos sertões: os povos indígenas no rio Branco e a colonização. Rio de Janeiro: Paz e Terra/AMPOCS.

FOCK, Niels. 1963. Waiwai, religion and society of an amazonian tribe. National Museum of Denmark, Ethnographic Series, 8.

FRIKEL, Protásio, fr. 1957. "Zur linguistisch-ethnologischen gliederung der indianerstämme von Nord-Pará (Brasilien) und den anliegenden Gebieten". Freiburg: Anthropos, vol. 52, (3-4): 509-63.

FUNAI (Fundação Nacional do Índio). 1978. "Wai-Wai". Revista de Atualidade indígena. Brasília, 2 (10) : 42-50

- 1987. Área indígena Trombetas/ Mapuera. Superintendência de Assuntos Fundiários (SUAF). [Manaus], 21 out. Mapa de interdição da área.

- 1989. "Estar allí. La antropología y la escena de la escritura". El antropólogo como autor. Barcelona: Paidos: 11-34.

GUPPY, Nicholas. 1958. Wai-Wai. London: John Murray.

HOWARD, Catherine V. 1985-6. "Procuramse almas". In: C. A. Ricardo (org.), Povos indígenas no Brasil. São Paulo: CEDI:126-127.

.1993. "Pawana: a farsa dos 'visitantes' entre os Waiwai da Amazônia setentrional". In: E. Viveiros de Castro \& M. Carneiro da Cunha (orgs.), Amazônia: etnologia e história indígena. São Paulo: NHII-USP:FAPESP.

MEGGERS, Betty J. 1977. Amazônia: a ilusão de um paraíso. Título original: Amazonia: man and culture in a counterfeit paradise. Chicago: Aldine, 1971; [Trad. de Maria Y. Linhares]. Rio de Janeiro: Civilização Brasileira. Col. Retratos do Brasil, v.100.

MEGGERS, Betty M. \& EVANS, C. 1964. "Genealogical and demographic information on the Wai Wai of Brit- ish Guiana". In: Beiträge zur Völkerkunde südamerikas. Völkerkundliche Abhandlungen (1): 199-207.

MENTORE, George P. 1984. Shepariymo: the political economy of the a Waiwai village. University of Sussex. Thesis.

. 1987. "Waiwai women: the basis of wealth and power". University of Virginia/Centro de Ivestigaciones Indigenas de Puerto Rico. Man, 22 (3): 511-527.

MILLER, Robert Pritchard. 1996 . "Movimentação Wai Wai". In: Carlos Alberto Ricardo (org.), Povos indígenas no Brasil 1991/95. São Paulo: Instituto Socioambiental: 256 .

MORTON, J. 1983-84. "Women as values, signs and power: aspects of the politics of ritual among the Waiwai". In: A. B. Colson \& H. D. Heinen (eds.), Themes in political organization: the caribs and their neighbours. Caracas: Fundatión La Salle, Antropológica, 59-62.

QUEIROZ, Ruben Caixeta de. 1996. "A saga de Ewká: Epidemia e evangelização entre os Wai Wai". In: Robin M. Wright (org.), Religiões indígenas e cristianismo no Brasil. Universidade Estadual de Campinas, Série Perspectivas Antropológicas, 1:214-241

RICARDO, Carlos Alberto (org.). 1996. "Waiwai". In: Povos indígenas no Brasil 1991/95. São Paulo: Instituto Socioambiental: 252-256.

RIVIÈRE, Peter. 1984. Individual and society in Guiana. Cambridge University Press.

1995. "Houses, places and people: community and continuity in Guiana". In: Janet Carsten \& Stephen Hugh-Jones (eds.), About the house: Lévi-Strauss and beyond. Cambridge University Press.

ROTH, Walter E. 1929. "Adtional studies of the arts, crafts, and customs of the Guiana indians". Washington. Bulletin, Bureau of America Ethnology, vol. 91. 
SCHOMBURGK, Richard. [1847-48] 1922-

23. Richard Schomburgk's travels in British Guiana. 2 vols. Georgetown.

TAUSSIG, Michael. 1993. Xamanismo, colonialismo e o homem selvagem: um estudo sobre o terror e a cura. Título original: Shamanism, Colonialism, and the wild man - A study in terror and healing. The University of Chicago, 1987; [Trad. de Carlos Eugênio M. de Moura]. Rio de Janeiro: Paz e Terra.

YDE, Jeans. 1965. Material culture of the WaiWai. Copenhagen: Natinalmuseets Skrifter, etnografisk Raekke X. 